\section{Análisis de la clorofila de spinacia oleracea y cuantificación de albumina de espagueti utilizando espectrofotometría*}

\section{Analysis of spinacia oleracea chlorophyll and quantification of albumin using spectrophotometry}

\author{
Jeisson Fabian Martin Calvo, ** Julián Castañeda Gómez***
}

**Doctorando en Enseñanza de las Ciencias mención Física.Profesor vinculación especial del Programa de Ingeniería Sanitaria, Facultad de Medio Ambiente y Recursos Naturales, Universidad Distrital Francisco José de Caldas (Bogotá, Colombia)

***Universidad Distrital Francisco José de Caldas (Bogotá, Colombia)

\section{Resumen}

Basándose en el fenómeno de pigmentación que se percibe en los objetos, el cual puede explicarse por análisis fisicoquímicos, en esta investigación se realiza la espectrofotometría ultravioletavisible (UV-VIS) para analizar la clorofila extraída de la Spinacia oleracea logrando interpretar y relacionar los conceptos de espectro de luz, absorbancia y pigmentos, mediante la elaboración y análisis de curvas de absorbancia. Por otro lado, se logra cuantificar las proteínas (albumina) extraída de pastas comestibles (espagueti) de diferentes costos, haciendo uso del método de Lowry que utiliza el reactivo de Folin, obteniendo de la evidencia experimental en estos casos, la relación de los términos de absorbancia y las concentraciones de diversas sustancias, mediante la ley de Lambert-Beer la cual se deduce matemáticamente.

Palabras clave: Absorbancia, albumina, clorofila, espectro de luz, spinacia oleracea, espectrofotometría, proteínas.

\begin{abstract}
Based on pigmentation analysis perceived in objects, which may be explained through physic-chemical analysis, the ultraviolet-visible (UV-VIS) spectrophotometry is realized in this research, in order to analyze the chlorophyll extracted from Spinacia oleracea, leading to interpret and relate the concepts of light spectrum, absorbance and pigments, through preparation and analysis of absorbance curves. In addition, proteins (albumin) extracted from different cost pasta (spaghetti), by using the Lowry method which uses Folin reagent, obtaining experimental evidence, in these cases the relationship of absorbance terms and concentrations of various substances, through Lambert-Beer's, which is mathematically deduced.
\end{abstract}

Key words: Absorbance, albumin, chlorophyll, light spectrum, spectrophotometry, proteins.
Recibido: 15/03/2016

Revisado: 26/04/2016

Aceptado: 10/12/2016

Correspondencia de autor:

jfmartinc@unal.edu.co juacastanedag@correo.udistrital. edu.co

(C) 2016 Universidad La Gran Colombia. Este es un artículo de acceso abierto, distribuido bajo los términos de la licencia Creative Commons Attribution License, que permite el uso ilimitado, distribución y reproducción en cualquier medio, siempre que el autor original y la fuente se acrediten.

Cómo citar:

Martín, J.F \& Castañeda, J. (2016) Análisis de la clorofila de spinacia oleracea y cuantificación de albumina de espagueti utilizando espectrofotometría.UGCiencia 22, 99-109.

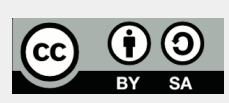




\section{Introducción}

La espectrofotometría UV-VIS es un protocolo experimental que facilita la identificación de concentraciones de diversas sustancias mediante la obtención de la absorbancia, por ende su importancia en temas de la salud pública prevalece, ya que entre sus diversas aplicaciones, se resaltan la cuantificación de contaminantes (Fresenius, Qentin, \& Scheneider, 2012), la evaluación de calidad de alimentos (Quezada Moreno, Gallardo, \& Torres, 2016), determinación de calidad de agua (Espinosa \& Rodriguez, 2016) y análisis de suelos (Rodríguez, García, \& Fajardo, 2016), por lo anterior es imprescindible exaltar la funcionalidad de dicho protocolo experimental con base a los principios de física y química que allí se contemplan.

El proceso que realiza la espectrofotometría UV-VIS inicia desde los colores que observa el ojo humano ya que es un fenómeno físico que describe la absorción de una parte del espectro de luz visible mientras otra se refleja o se transmite (Curtis, Schnek, \& Massarini, 2008); hasta la comprensión de la ley de Lambert-Beer la cual describe la relación que existe entre el fenómeno descrito anteriormente con la concentración de una substancia (Rastogi, 2010).

Partiendo de lo anterior, la presente investigación brinda un análisis pertinente acerca de la espectrofotometría mediante la relación que existe entre los conceptos de espectro de luz, absorbancia y pigmentos, en términos de la Ley de Lambert-Beer que se refiere a la absorbancia que originan los pigmentos, en este caso la clorofila extraída de Spinacia oleracea (espinaca).

Así mismo se presenta la utilización de la espectrofotometría mediante el método de Lowry para la cuantificación de proteínas presentes en tres tipos de pasta comestible (espagueti) de diferentes costos, para determinar su calidad en términos de concentración de proteínas con base a una muestra patrón de albumina. El estudio se realizó en el Laboratorio de Química, de la Facultad de Medio Ambiente y Recursos Naturales (FAMARENA) de la Universidad Distrital Francisco José de Caldas (Bogotá-Colombia).

Figura 1. Espectro de luz visible con sus respectivas medidas en nanómetros (Curtis, Schnek, \& Massarini, 2008).
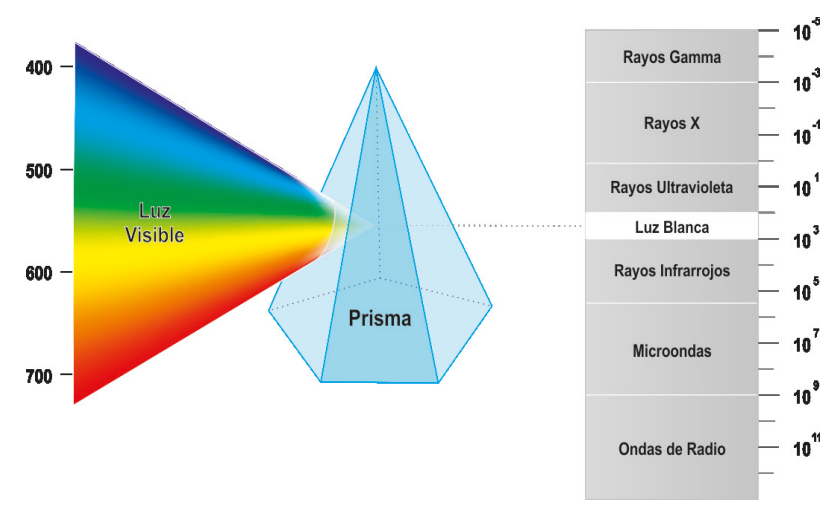

\section{Perspectiva teórica}

El espectro de luz visible es la descomposición de un rayo de luz proveniente del sol en diversos rangos determinados por colores con sus respectivas longitudes de ondas como se observa en la figura 1.

Cuando dichas longitudes de ondas inciden sobre un pigmento el color que se observa proviene del reflejo de dicho espectro mientras que alguno de los demás rangos es absorbido. Para entender este fenómeno es conveniente pensar en un rayo de luz proveniente del sol que incide en la hoja de una planta que posee clorofila ${ }^{1}$, el pigmento verde de todas las células

1. Las plantas verdes poseen clorofila y , además contienen otros pigmentos denominados carotenoides. Se diferencian por su composición molecular y cada 
fotosintéticas, cuyo papel en la fotosíntesis es indispensable debido a la captación de energía que toma de dicho rayo. En esta incidencia ocurre el reflejo del rango de espectro de luz proveniente del color verde que tiene una $\lambda$ de 500 a $600 \mathrm{~nm}$ de a mientras que otro rango del espectro es absorbido para diversas funciones, entre ellas, el fotosistema 1 y 2 (Nathan \& Yocum, 2006) ilustrados en la figura 2

En la figura 2 se ilustra como en el fotosistema II ocurre la fotolisis ${ }^{2}$ de mediante la excitación del ion magnesio ${ }^{3}$ presente en la estructura molecular de la clorofila que se observa en la figura 3. Esto tiene lugar por la energía brindada en la absorción de un fotón proveniente de la luz solar de . Luego, se libera un electrón que en el fotosistema I vuelve a recargarse de energía mediante la utilización de un fotón de .

Figura 2. Cadenado de transporte electrónico fotosintético mediante el fotosistema I y II (Curtis, Schnek, \& Massarini, 2008)

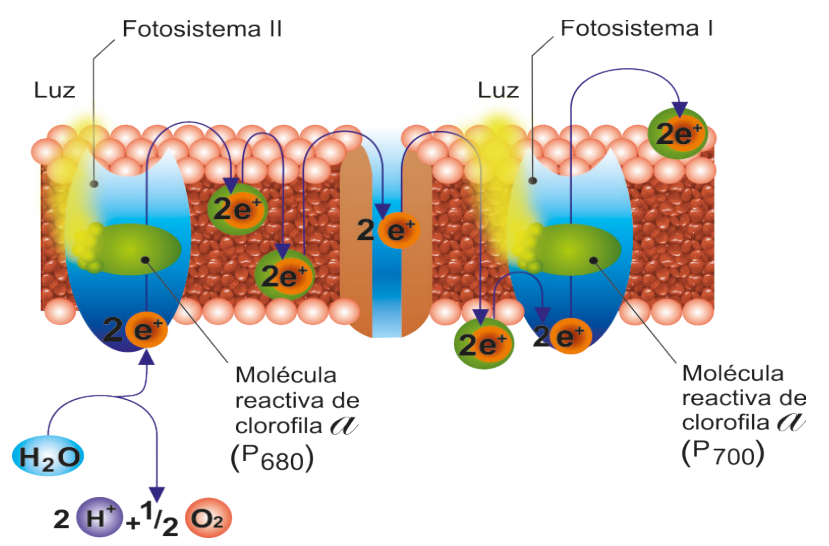

una tiene un papel importante en los procesos fotosintéticos, sin embargo, en esta investigación se enfatiza en la clorofila ya que es el pigmento que absorbe la energía lumínica y la transforma en energía química.

2. Ruptura causada por energía radiante de enlaces químicos.

3. La excitación del ion ocurre por la energía lumínica que absorbe, lo anterior genera la pérdida de un electrón de la molécula de la clorofila por lo tanto confiere una característica oxidativa tan grande que permite le fotolisis del agua.
Figura 3. Estructura molecular de la clorofila (Koolman \& Röhm, 2005).

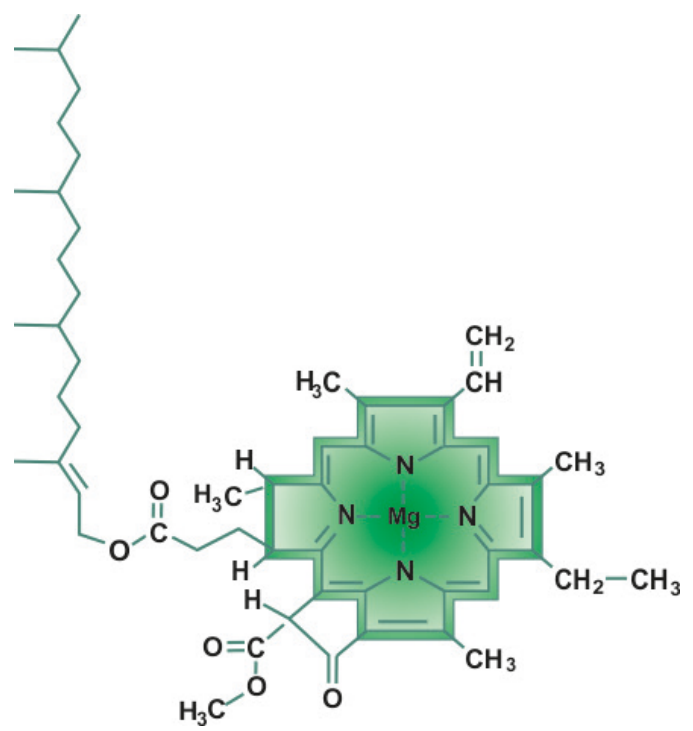

Con lo anterior se evidencia como el rango de absorción de energía presente en el color de la clorofila es de a. Lo cual es lógica ya que es un rango, que verificando la figura 1 , se debería absorber. Para determinar el anterior fenómeno en una sustancia, se utiliza el término de absorbancia mediante la Ley de Lambert-Beer. Como se muestra en la figura 4 cuando un haz de radiación monocromática paralela, de intensidad incide perpendicularmente sobre un bloque de materia, sólido, líquido o gas, capaz de absorber radiación, después de que el haz ha recorrido cierta trayectoria, , dentro de dicho bloque que contiene partículas capaces de absorber, ya sea átomos, iones o moléculas, su intensidad disminuye a un valor como consecuencia de la absorción de radiación, cabe resaltar que este es el proceso que realiza el espectrofotómetro.

Figura 4. Incidencia de un haz de luz de determinada, en una muestra problema. Siendo: 1) fuente de luz; 2), 4) y 6) rendijas; 3) monocromador; 5) cúbate con muestra de partículas; 7) registrador. 


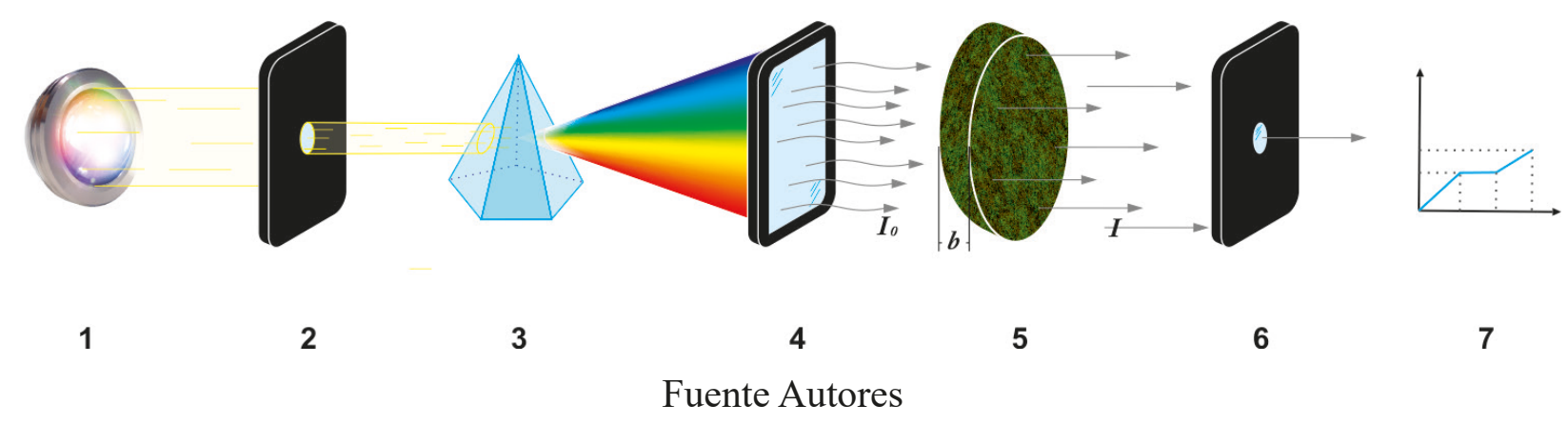

La fracción de disminución del haz de luz, al atravesar cada sección infinitesimal del material absorbente se puede representar en forma diferencial como _ $-\underline{d I}$, esta disminución es proporcional al I número de partículas absorbentes en cada sección transversal, de área, del material que atraviesa la radiación. La disminución total que sufre el haz de luz monocromático, después de atravesar la sustancia se representa mediante la expresión:

$$
-\int_{I_{0}}^{I} \frac{d I}{I}=\int_{0}^{n} \frac{a d n}{S}
$$

Donde es la constante de proporcionalidad llamada sección transversal de captura, debido a la relación directamente proporcional en cuanto a la disminución del haz incidente y al número de partículas absorbentes y el área de sección transversal de captura. Al solucionar la anterior expresión se obtiene:

$$
-\ln \left(\frac{I}{I_{0}}\right)=\left(\frac{a n}{s}\right)
$$

Aplicando $\ln \mathrm{x}=2.303 \log (\mathrm{x})$ y Área en términos de volumen $\mathrm{S}=\mathrm{V} / \mathrm{b}$ se tiene:

$$
-2.303 \log \left(\frac{I}{I_{0}}\right)=\left(\frac{a n b}{V}\right)
$$

Teniendo en cuenta la relación

$$
\text { moles }=\frac{n \text { (o numero de particulas) }}{6.02 \times 10^{23} \text { particulas } / \mathrm{mol}}
$$

el volumen en términos de litros

$$
V=\frac{V \mathrm{~cm}^{3}}{1000 \mathrm{~cm}^{3} / L}
$$

y la concentración

$$
c=\frac{1000 \mathrm{n}}{6.02 \times 10^{23} V}
$$

en la ecuación (2), la nueva expresión es:

$$
-\log \left(\frac{I}{I_{0}}\right)=\left(\frac{6.02 \times 10^{23} a b c}{2.303 \times 1000}\right)
$$

Donde Indica el Índice de absortividad, definida como la unidad de absorbancia por unidad de concentración por unidad de longitud de la trayectoria de la luz (Nobel, 2012), su expresión matemática es:

$$
\varepsilon=\frac{6.02 \times 10^{23} a}{2.303 \times 1000}
$$

Reemplazando (4) en (3) se obtiene:

$$
-\log \left(\frac{I}{I_{0}}\right)=\varepsilon . b . c
$$

En (5) ocurre el fenómeno de la transmitancia $T$ el cual es la relación de $I$ y $I_{0}$ y que pasa a través de una muestra, por lo tanto: $-\log (T)=$ E. b.c 
En términos de sustancias, la absorbancia , también conocida como Densidad Óptica, se define como la relación logarítmica de (Sogorb \& Vilanova, 2004), por ende:

$A=\varepsilon \cdot b \cdot c$

Para $\boldsymbol{b}$ en $[\mathrm{cm}], \boldsymbol{c}$ es la relación en $[\mathrm{mol} / \mathrm{L}], \mathrm{y} \mathcal{E}$ en $[\mathrm{mol} / \mathrm{L}]$

La ecuación (6) es la expresión de la Ley de Lambert-Beer, la cual indica la relación directamente proporcional que existe entre la absorbancia y la concentración de ciertas sustancias (Fresenius, Qentin, \& Scheneider, 2012). La espectrofotometría realiza el análisis de la absorbancia de acuerdo a la ley de Lambert-Beer y se realiza mediante el uso del espectrofotómetro.

\section{Materiales y métodos}

Este estudio contempla dos espectrofotometrías por UV-VIS e involucra el uso de las curvas de calibrado para la interpretación y discusiones de resultados, por lo tanto, se divide en dos fases de investigación: una realizada con la clorofila (Fase 1) y otra mediante la cuantificación de proteínas presentes en espagueti (Fase 2):

\section{Método de cuantificación de clorofila (Fase 1):}

Este método consta de extraer una cantidad significativa de clorofila $(500 \mathrm{ml})$ de 140 gr de Spinacia oleracea (espinaca) con la utilización de alcohol etílico y agua. Luego para cada caso, se realizaron las pruebas pertinentes con el espectrofotómetro mediante diferentes disoluciones de clorofila: 1,2 y 3 $\mathrm{ml}$, con la finalidad de obtener las respectivas absorbancias; como muestra patrón, para las espectrofotometrías se consideraron las muestras puras extraídas. Se realizaron 5 mediciones por espectrofotometría para cada concentración con el fin de establecer análisis pertinentes respecto al valor promedio en cada caso, tablas 1 y 2 .

\section{Método de Lowry para la cuantificación de proteínas (Fase 2):}

El procedimiento que se aplicó para la cuantificación de las proteínas es el método de Lowry, que utiliza el reactivo de Folin. En este método se desarrollan dos reacciones coloridas: primero se desarrolla un color de poca intensidad con el cobre, en este caso sulfato de cobre pentahidratado en medio alcalino, con lo que se forma un complejo de color azul. Por último, se realiza la reacción con el reactivo de Folin en la cual se forma un complejo de color verde para realizar las respectivas Espectrofotometrías (Trumbo, Schultz, Borland, \& Pugh, 2013).

La extracción de la proteína se realizó por medio de 3 tipos de pasta comestible teniendo en cuenta un precio comercial menor, medio y superior. Para ello se consideró 10 gr de cada tipo de pasta y se dejó en ebullición por $20 \mathrm{~min}$ en $250 \mathrm{ml}$ de agua, luego se filtraron en balones aforados, seguido a esto se utilizaron $3 \mathrm{ml}$ de las muestras y se procedió con la reacción del método de Lowry. Se realizaron 5 mediciones para cada caso (tanto para la muestra patrón como para la muestra problema) con el fin de establecer análisis pertinentes respecto al valor promedio en cada caso, tablas 3 y 4 .

La muestra patrón utilizada fue albumina pura teniendo en cuenta concentraciones de $0.3,0.6$ y 1.2 () proporcionada por el laboratorio de Química de la FAMARENA. La espectrofotometría UV-VIS se realizó con el fin de obtener las absorbancias de extracto de clorofila y de albumina para verificar la ley de Lambert-Beer y concentraciones utilizando el espectrofotómetro spectroquant pharo 100. 


\section{Curva de calibrado:}

Con el fin de cuantificar y verificar gráficamente los datos de absorbancias se obtiene la curva de calibrado midiendo la absorbancia de una serie de soluciones de concentraciones conocidas de una misma sustancia, tratadas con un mismo método y medidas a igual longitud de onda en el mismo instrumento. Para medir la concentración de una sustancia se eligió la región de máxima absorción del espectro con base a la muestra patrón, denominada longitud de onda analítica: En el caso de la Fase 1 se optó un intervalo de de a, realizando mediciones de espectrofotometría de la muestra patrón ascendentes de 10 en 10 y de 5 en 5 , con el fin de obtener graficas bidimensionales (Absorbancia vs ) para evidenciar las máximas de absorbancia en las espectrofotometrías correspondientes, así mismo se realizó el cálculo de las concentraciones mediante la constante de la concentración por el volumen ().

Teniendo en cuenta los valores promedios para cada caso (Extracción por alcohol y por agua), se obtuvo la correlación lineal () para el modelo que mejor se ajuste entre el lineal, logarítmico o exponenciales, la Raíz del Error Medio Cuadrático (REMC) y el porcentaje de confianza.

Para la fase 2 se realizó un proceso análogo respecto a la fase 1en cuanto a la identificación de la máxima absorbancia del espectro con un intervalode dea, porotroladomediantelosvalores promedio obtenidos de las espectrofotometrías, se obtuvieron las concentraciones desconocidas mediante una interpolación de datos teniendo en cuenta y el modelo de correlación más pertinente, la REMC y el porcentaje de confianza.

\section{Resultados y discusión}

A continuación se presentan los resultados obtenidos en la Fases 1y 2 y sus respectivos análisis.
En el primer caso se resaltan los datos obtenidos con la clorofila extraída con alcohol etílico y agua; en el segundo se plantean los resultados de la cuantificación de proteínas teniendo en cuenta una muestra patrón.

\section{Resultados de la espectrofotometría de la clorofila (Fase 1)}

Resultados de la espectrofotometría de clorofila extraída con alcohol etílico

Tabla 1. Resultados de las absorbancias registradas por el espectrofotómetro y el valor promedio de los datos, teniendo en cuenta el registro de la muestra patrón de por el espectrofotómetro en el caso de Clorofila extraída con alcohol etílico.

\begin{tabular}{|c|c|c|c|c|c|c|}
\hline \multirow{2}{*}{$\begin{array}{c}\text { Concentración } \\
(\mathrm{ppm})\end{array}$} & \multicolumn{5}{|c|}{ Mediciones (absorbancias) } & \multirow{2}{*}{ Promedio } \\
\cline { 2 - 6 } & 1 & 2 & 3 & 4 & 5 & \\
\hline 56000 & 0,073 & 0,075 & 0,076 & 0,074 & 0,076 & 0,075 \\
\hline 112000 & 0,194 & 0,174 & 0,204 & 0,230 & 0,218 & 0,204 \\
\hline 168000 & 0,255 & 0,245 & 0,234 & 0,268 & 0,274 & 0,255 \\
\hline
\end{tabular}

Resultados de la espectrofotometría de clorofila extraída con agua

Tabla 2. Resultados de las absorbancias registradas por el espectrofotómetro y el valor promedio de los datos. Teniendo en cuenta el registro de la muestra patrón de por el espectrofotómetro en el caso de Clorofila extraída con agua.

\begin{tabular}{|c|c|c|c|c|c|c|}
\hline \multirow{2}{*}{$\begin{array}{c}\text { Concentración } \\
(\mathrm{ppm})\end{array}$} & \multicolumn{5}{|c|}{ Mediciones (absorbancias) } & \multirow{2}{*}{ Promedio } \\
\cline { 2 - 7 } & 1 & 2 & 3 & 4 & 5 & \\
\hline 56000 & 0,088 & 0,107 & 0,098 & 0,115 & 0,126 & 0,107 \\
\hline 112000 & 0,153 & 0,146 & 0,238 & & 0,266 & 0,201 \\
\hline 168000 & 0,238 & 0,294 & 0,279 & & 0,313 & 0,279 \\
\hline
\end{tabular}


Discusiones de resultados para el caso de la clorofila:

A continuación, se presentan las discusiones de los resultados de la fase 1 mediante la interpretación y elaboración de las curvas de calibrado de las absorbancias obtenidas mediante la espectrofotometría UV:

Curva de calibrado obtenida en el caso de la clorofila extraída con alcohol etílico (Tabla 1):

Gráfica 1. Curva de calibrado teniendo en cuenta que la abscisa comprende la concentración de clorofila extraída con alcohol etílico y el eje de ordenadas se refiere a la absorbancia obtenida por la espectrofotometría.

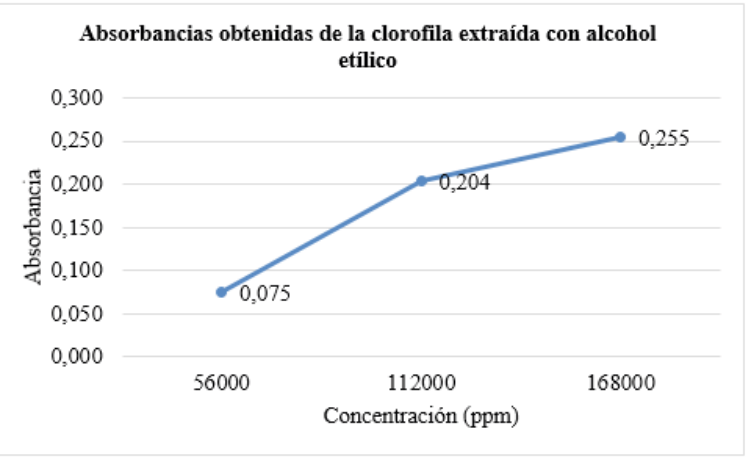

La gráfica 1 representa los valores promedio de las absorbancias obtenidas por espectrofotometría UV y presenta un de 0.9955 para un modelo logarítmico ${ }^{4}$, un REMC de 0.0071 y una confianza promedio de $93.6 \%$ con lo cual se comprende una obtención de datos optima y una elaboración de curva de calibrado pertinente para establecer concentraciones desconocidas, en este caso, de clorofila extraída con alcohol etílico.

Curva de calibrado obtenida en el caso de la clorofila extraída con agua, Tabla 2:

4. Siendo la absorbancia y la concentración en
Gráfica 2. Curva de calibrado teniendo en cuenta que la abscisa comprende la concentración de clorofila extraída con agua y el eje de ordenadas se refiere a la absorbancia obtenida por la espectrofotometría.

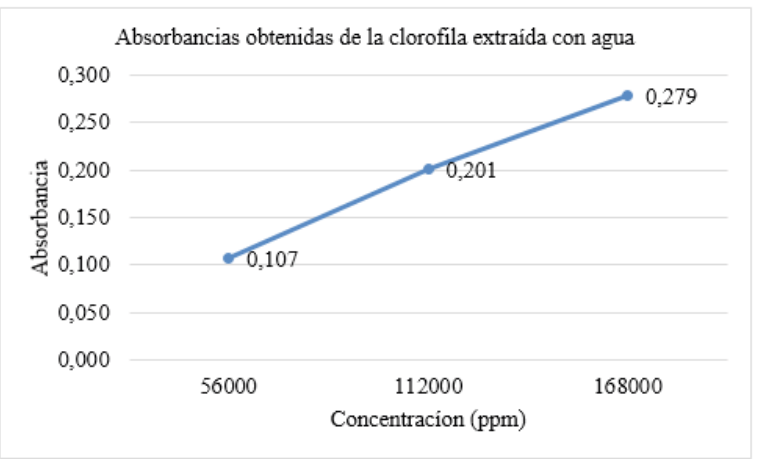

La gráfica 2 representa los valores promedio de las absorbancias obtenidas por espectrofotometría UV y presenta un de 0.9986 para un modelo lineal ${ }^{5}$, un REMC de $0.0202 \mathrm{y}$ una confianza promedio de $83.4 \%$ con lo cual se comprende una obtención de datos optima y una elaboración de curva de calibrado pertinente para establecer concentraciones, en este caso, de clorofila extraída con agua.

Se evidencia que las estadísticas obtenidas en gráfica 1 ofrecieron mejores resultados respecto a la gráfica 2 , lo anterior es debido al método de extracción porque el primer caso (extracción con alcohol etílico) presenta una afinidad química con la molécula de clorofila ya que se trata de un solvente apolar.

\section{Resultados de la espectrofotometría de la albumina (Fase 2)}

A continuación se presentaran los resultados obtenidos en la fase 2 teniendo en cuenta los datos obtenido por la espectrofotometría UV de la muestra patrón, para posteriormente evidenciar la cuantificación de proteínas

5. Siendo la absorbancia y la concentración en 
presentes en los espaguetis seleccionados mediante la interpolación de datos:

Resultados obtenidos cuantitativamente de la muestra Patrón:

Tabla 3. Resultado de las absorbancias registradas por el espectrofotómetro y el valor promedio de los datos para el caso de la muestra Patrón.

\begin{tabular}{|c|c|c|c|c|c|c|}
\hline \multirow{2}{*}{$\begin{array}{c}\text { Concentración } \\
(\mathrm{mg} / 1000 \mathrm{ml})\end{array}$} & 1 & 2 & 3 & 4 & 5 & \multirow{2}{*}{ Promedio } \\
\cline { 2 - 6 } & 0,292 & 0,314 & 0,304 & 0,274 & 0,276 & 0,292 \\
\hline 0,3 & 0,514 & 0,520 & 0,509 & 0,557 & 0,500 & 0,520 \\
\hline 0,6 & 0,699 & 0,706 & 0,730 & 0,722 & 0,752 & 0,722 \\
\hline 1,2 & & \multicolumn{4}{|c|}{} & \\
\hline
\end{tabular}

Resultados obtenidos de las muestras de espaguetis siendo en cuanto a precios comerciales:

Tabla 4. Resultados de las absorbancias registradas por el espectrofotómetro y el valor promedio de los datos. Las concentraciones se obtuvieron mediante la interpolación de datos respecto a la prueba patrón, usando un modelo logarítmico ${ }^{6}$ ya que el valor de obtenido de es de 0,9994 y REMC de 0,0061 .

\begin{tabular}{|c|c|c|c|c|c|c|c|}
\hline \multirow[b]{2}{*}{$\begin{array}{c}\text { Mue. } \\
\text { problema }\end{array}$} & \multirow{2}{*}{$\begin{array}{c}\text { Concen- } \\
\text { tración } \\
(\mathrm{mg} / 1000 \mathrm{ml})\end{array}$} & \multicolumn{5}{|c|}{ Mediciones (absorbancias) } & \multirow{2}{*}{ Prom. } \\
\hline & & 1 & 2 & 3 & 4 & 5 & \\
\hline $\mathrm{M}_{1}$ & 1,152 & 0,720 & 0,720 & 0,714 & 0,708 & 0,707 & 0,714 \\
\hline $\mathrm{M}_{2}$ & 0,679 & 0,583 & 0,538 & 0,529 & 0,550 & 0,550 & 0,550 \\
\hline $\mathrm{M}_{3}$ & 0,981 & 0,655 & 0,664 & 0,676 & 0,673 & 0,650 & 0,664 \\
\hline
\end{tabular}

6

Siendo la absorbancia y la concentración en
Discusiones de resultados para el caso de las proteínas:

A continuación, se presentan las discusiones de los resultados de la fase 2 mediante la interpretación y elaboración de las curvas de calibrado de las absorbancias obtenidas mediante la espectrofotometría UV tanto para la muestra patrón como para la muestra problema. Finalmente se presenta en un solo gráfico dichas curvas para interpretar una discusión general de la fase 2:

Teniendo en cuenta los valores promedios de las absorbancias obtenidas de la tabla 3, la correspondiente curva de calibrado de la prueba patrón respectivamente es:

Gráfica 3. Curva de calibrado teniendo en cuenta que la abscisa comprende la concentración de Proteína (albumina) y el eje de ordenadas se refiere a la absorbancia obtenida por la espectrofotometría.

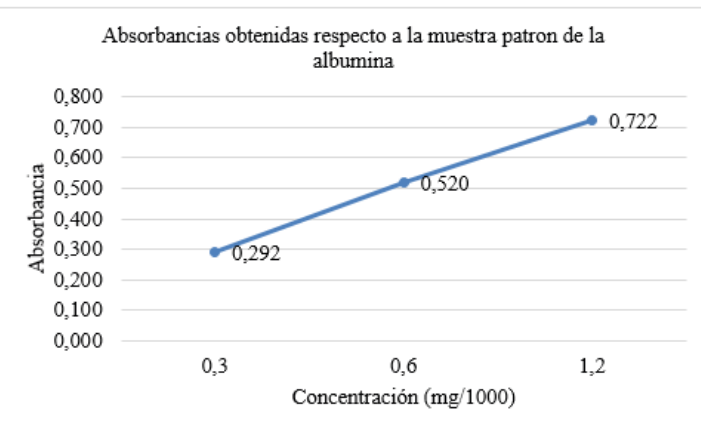

Como se observa en la gráfica 4 el orden, de concentración de albumina presente en las muestras desconocidas de espaguetis, fue , esto se debe a la presencia de otras proteínas presentes en el espagueti que participan como trazas o sustancias adicionales respecto a la muestra patrón de albumina. 
Gráfica 4. Curva de calibrado de la prueba de la muestras teniendo en cuenta que la abscisa comprende la concentración de Proteína y el eje de ordenadas se refiere a la absorbancia obtenida por la espectrofotometría.

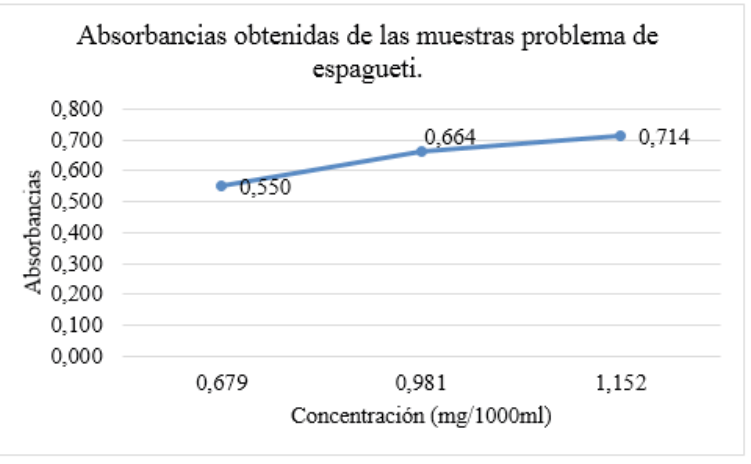

Contrastando las gráficas 3 y 4 se obtiene la gráfica 5 donde se observa un comportamiento directamente proporcional a las proteínas, es decir, en cuanto a la concentración de proteínas aumenta, la absorbancia también lo hace:

Gráfica 5. Curva de calibrado general de albumina pura y extraída de espaguetis teniendo en cuenta que la abscisa comprende la concentración de Proteína y el eje de ordenadas se refiere a la absorbancia obtenida por la espectrofotometría.

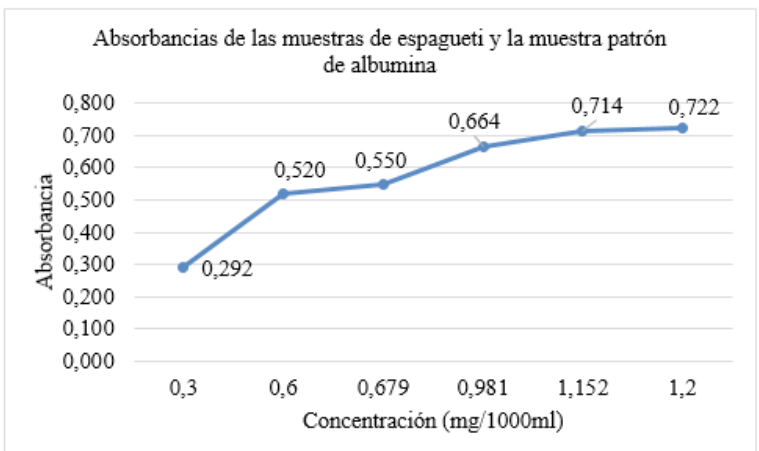

La gráfica 5 presenta un de 0.9996 para un modelo logarítmico ${ }^{7}$, un REMC de 0.004 y una confianza de $72 \%$ con lo cual es permisible realizar interpolaciones óptimas para calcular concentraciones respecto a la obtención de absorbancias, así mismo muestra la importancia del método de Lowry para concluir condiciones de calidad de alimentos ya que, en este caso, de diversas pastas con precios comerciales diferentes se logra la cuantificación de proteínas mediante una muestra patrón con unas concentraciones precisas de albumina.

\section{Discusiones generales}

La gráfica 2 presenta un comportamiento lineal más notable que la gráfica 1 , esto se debe posiblemente a las trazas presentes por la extracción de la sustancia ya que molecularmente se presenta más absorbancia en algunas regiones de la muestra. Contrastando ambas graficas observamos un comportamiento directamente proporcional, es decir que en cuanto a la concentración de clorofila aumenta, la absorbancia también lo hace. Así mismo, cabe resaltar la longitud de onda registrada por el espectrofotómetro en ambos casos para la muestra patrón (véase las leyendas de las tablas 1 y 2) ya que teóricamente ambos valores están en el rango de espectro de luz óptimo de absorbancia.

En el caso de la gráfica 5 se evidencia una confianza de $72 \%$ lo cual es ligeramente bajo y su valor es debido a la extracción general de proteínas que el método de Lowry ofrece por lo tanto en las concentraciones obtenidas también se contemplan trazas de otras proteínas que contienen las pastas, sin embargo en dado caso que no fuese albumina y existiese otro tipo de proteínas, no se hubiese podido establecer un interpolación de datos sino una extrapolación de los mismos ya que la concentración obtenida hubiese sido mayor y por ende la absorbancia seria considerablemente alta respecto a la muestra patrón de albumina pura. 
Al analizar las gráficas 1, 2, 3, 4 y 5 se demuestra experimental la ley de Lambert-Beer, al evidenciar un comportamiento de absorbancia directamente proporcional a la concentración de una sustancia, en este caso de clorofila y albumina.

\section{Conclusiones}

El protocolo experimental de la Espectrofotometría UV, en primera instancia, se debe contemplar desde una perspectiva físico-química, para posteriormente entender métodos que involucren el uso del mismo, por lo tanto se presentan las fases 1 y 2 , para contemplar la relación teórico-práctica que representa dicho protocolo. Lo anterior se evidencia por la relación de la absorbancia respecto a los pigmentos y al espectro de luz visible obtenidos en el apartado Resultados y Discusiones de la fase 1 (componente teórico), así mismo, se muestra la interpretación de la fase 2 como un método que involucra el mismo principio pero aplicado en el análisis de alimentos (componente practico o experimental).

Se demostró experimentalmente para la fase $1 \mathrm{y}$ 2 que la absorbancia tiene una magnitud mayor en cuanto a la concentración aumenta, es decir, es directamente proporcional a la concentración que se posee en una muestra, esto tiene lugar por la ley de Beer la cual indica que a mayor concentración las ondas electromagnéticas chocan contra un mayor número de átomos o moléculas y son absorbidos, en cuanto a la fase 1 debido al protocolo de extracción y en la fase 2 por la presencia de trazas o sustancias adicionales.

Se demostró cuantitativamente que la energía absorbida corresponde a la cantidad necesaria para promover un electrón de un orbital a otro en una molécula conjugada, esto tiene lugar ya que la espectrofotometría fue realizada con radiación UV y la región máxima de absorbancia registrada por el fotómetro para el caso de la muestra patrón de la fase 1 estuvo en el rango teórico resaltado en la perspectiva teórica.

La espectrofotometría UV-VIS realizada en esta investigación para la cuantificación de albumina (fase 2) demuestra que el espagueti de menor precio presenta una mayor absorbanciay porende una mayor concentración no solo de albumina sino de otras proteínas que en ocasiones no son benéficas para los consumidores.

\section{Referencias bibliográficas}

Curtis, H, Schnek, A, \& Massarini, A. (2008). Curtis. Biología. En H. Curtis, A. Schnek, \& A. Massarini, Curtis. Biología. Buenos Aires: Editorial Médica Panamericana.

Espinosa, T, \& Rodriguez, C. (2016). Nota tecnica: Determinación del índice de calidad del agua (ICA), de los ríos Moron y Patanemo del Estado Carabobo, en Venezuela. Ingeniería uc, 206-213.

Fresenius, W, Qentin, K, \& Scheneider, W. (2012). Water Analysis, A practical guide to Physico-Chemical, Chemical and Microbiological water examination and Quality Assurance. En W. Fresenius, K. Qentin, \& W. Scheneider, Water Analysis, A practical guide to Physico-Chemical, Chemical and Microbiological water examination and Quality Assurance. Springer Science \& Business Media. 91-92.

Koolman, J, \& Röhm, K.-H. (2005). Bioquímica: texto $\mathrm{y}$ atlas. En J. Koolman, \& K.-H. Röhm, Bioquímica: texto y atlas. Ed. Médica Panamericana, 129-130. 
Nathan, N, \& Yocum, C. (2006). Stucture and function of photosystems 1 and 11. Annual Review of Plant Biology, 521-565.

Nobel, P. S. (2012). Physicochemical and Plant Physiology. En P. Nobel, Physicochemical and Plant Physiology San Diego California: Academic Press Limited, 228-234.

Quezada Moreno, W, Gallardo, I, \& Torres, M. (2016). El color en la calidad de los edulcorantes de la agroindustria panelera. Afinidad, 27-29.

Rastogi, S. C. (2010). Biochemistry. En S. C. Rastogi, Biochemistry (págs. 697-702). New Delhi: Trata McGraw Hill Education Private Limited.
Rodríguez, W, García, P, \& Fajardo, A. (2016). Aplicaciones de Técnicas Espectroscópicas para el Análisis de Suelos. Revista: Facultad de ciencias básicas, 228-243.

Sogorb, M, \& Vilanova, E. (2004). Técnicas analíticas de contaminantes químicos: Aplicaciones toxicológicas, medioambientales y alimentarias. En M. Sogorb, \& E. Vilanova, Técnicas analíticas de contaminantes químicos: Aplicaciones toxicológicas, medioambientales y alimentarias (págs. 85-86). Ediciones Díaz de Santos.

Trumbo, T, Schultz, E, Borland, M, \& Pugh, M. (2013). Applied Spectrophotometry: Analysis of a Biochemical Mixture. Laboratory Exercise, 242-250. 